\title{
Spatial Map Data Share and Parallel Dissemination System Based on Distributed Network Services and Digital Watermark
}

\author{
Dong Zhang ${ }^{1,2}$, Depei Qian ${ }^{1}$, Weiguo $\mathrm{Wu}^{1}$, Ailong $\mathrm{Liu}^{2}$, \\ Xuewei Yang ${ }^{2}$, and Pen $\mathrm{Han}^{2}$ \\ ${ }^{1}$ Department of computer science, Xi' an Jiaotong University, Xi' an, China \\ Navgride163.com \\ ${ }^{2}$ Xi' an Research Institute of Surveying and Mapping, Xi'an, China
}

\begin{abstract}
A distributed map data parallel dissemination system, which can be used to distribute spatial map data to remote users through different networks, is presented. The paper first defines spatial map data, associated metadata and network resource nodes with graphic formalization definition. Then, the relations between map data and network resources are established. Based on formalized definition to the whole system, a map data dissemination framework and a series of essential distributed CORBA services, conditions input methods based on three querying conditions are presented. We also explore network map copyright validating in dissemination course, and present an improved adaptive watermarking algorithm for vector digital maps whose data is the most difficult and necessary to watermark. Different from other methods, we compare the watermarked map with the original watermarked map, not with the primitive map, before extraction. The results show that compared with Direct Copy, our dissemination system can delivery spatial map data effectively. The transfer performance of CORBA services mode is almost equal to Direct Copy. The system can approach the biggest transfer rate more quickly but dependents on data amount weakly. And, the improved watermarking algorithm has better robustness.
\end{abstract}

Keywords: Map data, Metadata, Network services, Watermarking.

\section{Introduction}

The Map Data Network Dissemination system (MDND) is dissemination of Spatial Map Data (SMD) to remote users under network environment. The SMD data have such characteristics as storage in distributed form, high precision, mass data, multiscales, which is suitable to be applied in the network environment [1]. Meanwhile, network has become the main mean to obtain SMD today and in future. The statistics data in developed countries show that more than half information search is correlative to spatial position. However, due to limited resources and developing technical means, the map data dissemination services through network lie in a relatively lower level in China. There are several technical issues lied in SMD dissemination process. 
They are SMD and map metadata management and application modes, network services design and configuration, whole data security technique, network transferring course and user ends [2]. Data security involves many research subjects. In this paper, we mainly research copyright validating algorithm being essential in dissemination. As the primary copyright validating approach, watermarking technology is difficulty and emphases, especially for vector map data focused in relative research fields.

\section{Review of Correlative Technologies}

Special dissemination systems in main developed countries have been realized and, some of them in America and England have been used in real dissemination although with simple functions and narrow application fields. Not only in developed countries, but also in developing countries, map dissemination systems are researched widely. For example, Surveying and Mapping Authority of the Republic of Slovenia has done a lot of work in the field of elaboration of the reference data sets [3].

In china, however, research on special map data dissemination system supporting all map types has just begun. Although there are vast map applications cases via internet, the map data type and quantity in the cases are limited and the area is always local.

At the same time, SMD is difficult to be processed in real time and employed in real demands from remote server nodes effectively without special dissemination system. Metadata is essential in remote SMD data access [4].

A static map may exist as a predefined image or a customized image created by a CGI Server-side utility program [5]. [6] has analyzed distributed module service technology. [7] studied data sharing and visualization based on ActiveX and Dcom. [8] gave out a JAVA network map access and display engine. [9] presented a method to implement data share by using Web Service frame. As a whole, the disadvantages through above technologies to implement SMD share and dissemination lie in the following aspects, which make a special dissemination system in urgent need:

(1) Weak for complex computation, high precision and discrete distribution;

(2) Weak for virtual computation, 3-D visualization in SMD dissemination;

(3) Limited data types;

(4) Low repetitive use efficiency for software codes and resources.

In network dissemination system, digital map copyright could not be ignored. Digital watermarking is definitely one of the most successful solutions. Vector maps, the most important type in SMD, are constructed by coordinates of points, lines and polygons whose format have the characteristic of flow data not raster data [10]. The algorithms for vector maps need to consider the robustness under revisions. Therefore, most classic watermark embedding and detection algorithms could not be applied directly to vector maps [11]. Probably due to these difficulties, we know of only a few published works on watermarking of vector digital maps. [12] subdivided a vector map into some rectangular blocks of adaptive sizes according to the density of vertices and embedded each bit by displacing the vertices in a block. [13] and [14] proposed the Fourier descriptors for watermarking of graph images. [15] divided a vector map into rectangular blocks with the uniform area. 
We have constructed an experimental MDND system based on CORBA Visibroker framework and an improved adaptive watermarking algorithm for vector digital maps.

\section{Dissemination Method}

\subsection{SMD Resources in Network Environment}

Definition 1: Network Resources (NR) is defined as a relax Graph, $G=(N, A)$, where $\mathrm{N}$ is the network nodes set classified as SMD resources nodes $\mathrm{N}_{\mathrm{r}}$ and metadata information nodes $\mathrm{N}_{i}$, and $\mathrm{A}$ is the connections set. $|\mathrm{N}|=\mathrm{n}$ ' is the nodes number, and the $\mathrm{i}^{\text {th }}$ node is represented by $\mathrm{n}_{\mathrm{i}}$, and the $\mathrm{W}-\mathrm{n}_{\mathrm{i}}$ is the weight of time cost for searching $n_{i} \cdot|A|=m$ is the sides number, and the $i^{\text {th }}$ side is represented by $\operatorname{arc}_{i}$, and the $\mathrm{W}$-arc ${ }_{\mathrm{i}}$ is the weight of time cost for searching $\operatorname{arc}_{\mathrm{i}}$.

Definition 2: $\mathrm{R}$ stands for network resources $\mathrm{NR}$, and $|\mathrm{R}|$ represents the resources number. We define the relations between resources $r$ and resources nodes $\left(n_{i}\right)$ as In $\left(\mathrm{n}_{\mathrm{i}}, \mathrm{r}\right), \mathrm{n}_{\mathrm{i}} \in \mathrm{N}_{\mathrm{r}}, \operatorname{In}\left(\mathrm{n}_{\mathrm{i}}, \mathrm{r}\right)=1$ represents $\mathrm{r}$ is on the resource node $\mathrm{n}_{\mathrm{i}}$.

Definition 3: SMD Metadata is classified to six classes such as MRas, MIma, MVec, MEle, MBen and MGeo, which present the metadata of raster, image, vector, elevation, benchmark and geodesy orderly. Simply they are presented as $\mathbf{M}_{s u b}^{i}$. We define content entity and element set as follows. SVec $(\mathrm{k}, \mathrm{r})$ represents the element $\mathrm{r}$ of entity $\mathrm{k}$ in MVec.

$$
M_{e n t}=\{\operatorname{SRas}(\mathrm{k}, \mathrm{r}), \operatorname{SIma}(\mathrm{k}, \mathrm{r}), \operatorname{SVec}(\mathrm{k}, \mathrm{r}), \operatorname{SEle}(\mathrm{k}, \mathrm{r}), \operatorname{SBen}(\mathrm{k}, \mathrm{r}), \mathrm{SGeo}(\mathrm{k}, \mathrm{r})\}
$$

Definition 4: Define Metadata base M-DB as (2). Sub-databases $\mathrm{B}_{M}^{i}$ can be defined as (3) corresponding to $\mathrm{M}_{s u b}^{i}$ and data dictionary $\mathrm{B}_{i}{ }_{i}$. The database establishment rules are denoted as $\mathrm{C}_{\mathrm{i}}$. Here, $\mathrm{B}_{i}$ is relative to comparison table of data, data field, and other tables.

$$
\begin{gathered}
\mathrm{BM}=\{\text { BRas, BIma, BVec, BEle, BBen, BGeo }\} \\
\mathrm{B}_{M}^{i}=\mathrm{C}_{\mathrm{i}}\left(\mathrm{M}_{\text {sub }}^{i}, \mathrm{~B}_{i}^{\prime}\right)
\end{gathered}
$$

Definition 5: The relationships among metadata-set, metadata-base and information nodes $\mathrm{n}_{\mathrm{i}}$ : Logically, Set $\left(\mathrm{B}_{M}^{i}\right)$, including one or several $\mathrm{B}_{M}^{i}$, is defined as a network information node $\mathrm{n}_{\mathrm{i}} \in \mathrm{N}_{r}$, here $\mathrm{In}\left(\mathrm{n}_{\mathrm{i}}, \mathrm{r}\right)=1$, and $\mathrm{r}=\operatorname{Set}\left(\mathrm{B}_{M}^{i}\right)$. 


\subsection{MDND and CORBA Distributed Services}

According to method for entity and relation, define $\mathrm{D}=\left(\mathrm{E}, \mathrm{R}^{\prime}\right)$, as follows:

Definition 6: The entity of MDND is a five-elements set. Here, U, BM, B, c, s stand respectively for users, metadata and SMD base, query condition, and network service.

$$
\mathrm{E}=\{\mathrm{U}, \mathrm{BM}, \mathrm{B}, \mathrm{c}, \mathrm{s}\}
$$

Definition 7: The relations in MDND are $\mathrm{R}^{\prime}=\left\{\mathrm{R}_{\text {sub }}^{i}, 1 \leq \mathrm{i} \leq 6\right\}, \mathrm{R}_{\text {sub }}^{i}$ represents the all relations within entities. How to design the correlative services depends on MDND entity relations. Several entity relations and services are designed as Table 1. It should be pointed out that the given services S3-S10 (S4 exception) are formalization services, which must be changed to different service contents due to different data types [16].

There are also many other absolute computations in the system called absolute services and loosely connected with network, shown in Table 2.

Table 1. Corresponding table between entity relations and distributed services

\begin{tabular}{|c|c|c|c|c|}
\hline $\mathrm{R}^{\prime}$ & $\begin{array}{l}\text { Entity/ } \\
\text { relation }\end{array}$ & Meaning & $\begin{array}{lcl}\text { IDL definition for } \\
\text { CORBA Service }\end{array}$ & Service content \\
\hline $\mathrm{R}_{\text {sub }}^{1}$ & $(\mathrm{U}, \mathrm{c})$ & $\begin{array}{l}\text { Acquisition } \\
\text { term input }\end{array}$ & $\begin{array}{l}\text { S1:UserManagment() } \\
\text { S2:IORService() }\end{array}$ & $\begin{array}{l}\text { User management service } \\
\text { IOR service }\end{array}$ \\
\hline $\mathrm{R}_{\text {sub }}^{2}$ & $(\mathrm{c}, \mathrm{BM})$ & For metadata & S3:Metalocate() & Search for metadata-bases \\
\hline $\mathrm{R}_{s u b}^{3}$ & $(\mathrm{BM}, \mathrm{s})$ & $\begin{array}{l}\text { Metadata bases } \\
\text { services }\end{array}$ & $\begin{array}{l}\text { S4:RightPro() } \\
\text { S5:MetaDataQuery() } \\
\text { S6:MetaDataGet() }\end{array}$ & $\begin{array}{l}\text { Data authorization service } \\
\text { Metadata searching service } \\
\text { Metadata acquisition service }\end{array}$ \\
\hline $\mathrm{R}_{s u b}^{4}$ & $(\mathrm{BM}, \mathrm{B})$ & $\begin{array}{l}\text { SMD bases } \\
\text { location }\end{array}$ & S7:Maplocate() & SMD route pointing service \\
\hline $\mathrm{R}_{\text {sub }}^{5}$ & $(\mathrm{~B}, \mathrm{~s})$ & $\begin{array}{l}\text { SMD bases } \\
\text { distributed } \\
\text { services }\end{array}$ & $\begin{array}{l}\text { S4: RightPro() } \\
\text { S8:DataQuery() } \\
\text { S9:DataPreDisplay() } \\
\text { S10:DataGet() } \\
\end{array}$ & $\begin{array}{l}\text { SMD contents searching } \\
\text { Pre-display and confirming } \\
\text { SMD acquisition service }\end{array}$ \\
\hline $\mathrm{R}_{\text {sub }}^{6}$ & $(\mathrm{U}, \mathrm{B})$ & DownloadSMD & S11:DailyRecord() & Log service \\
\hline
\end{tabular}

Table 2. Absolute services table

\begin{tabular}{cl}
\hline $\begin{array}{l}\text { Absolute Service in Servers } \\
\text { (before transferred from servers) }\end{array}$ & $\begin{array}{l}\text { Absolute Service in Client } \\
\text { (after received on clients) }\end{array}$ \\
\hline & S16:DataDe-compression() \\
S12:DataCompression() & S17:DataDe-encrypting() \\
S13:DataEncrypting() & S18:WatermarkDetection() \\
S14:WatermarkEmbedding() & S19:DataBackuping() \\
S15:DataEncapsulation() & S20:CoherenceChecking() \\
& S21:CacheManagement() \\
& S22:DisplayEngine() \\
\hline
\end{tabular}




\subsection{MDND Architecture Based on Distributed Services}

The dissemination system architecture based on CORBA ORB is shown as Fig. 1. Here, only a part of main data operation services, which can reflect dissemination flow, are given. Metadata and SMD are distributed in server ends. For SMD, it is logically comprised of several bases according to the data types. The SMD Data operation services include data query, pre-display, acquisition of S8-S10 and authorization service S4. Moreover, a series of complex algorithms without close relations with network operations, such as data encryption, data compression, watermarking, etc, are designed as absolute services, which are essential to dissemination. Similar to SMD, metadata is supported mainly by the data query service S5, the acquisition service S6 and the authorization S4.

The client end shows the whole dissemination flow, including logical demands presentation, data de-compression and de-encryption, watermark detection, pre-display and display of metadata and SMD, user and log management (S11, S16-22), etc. This paper mainly research network operation services and absolute services S14, S18.

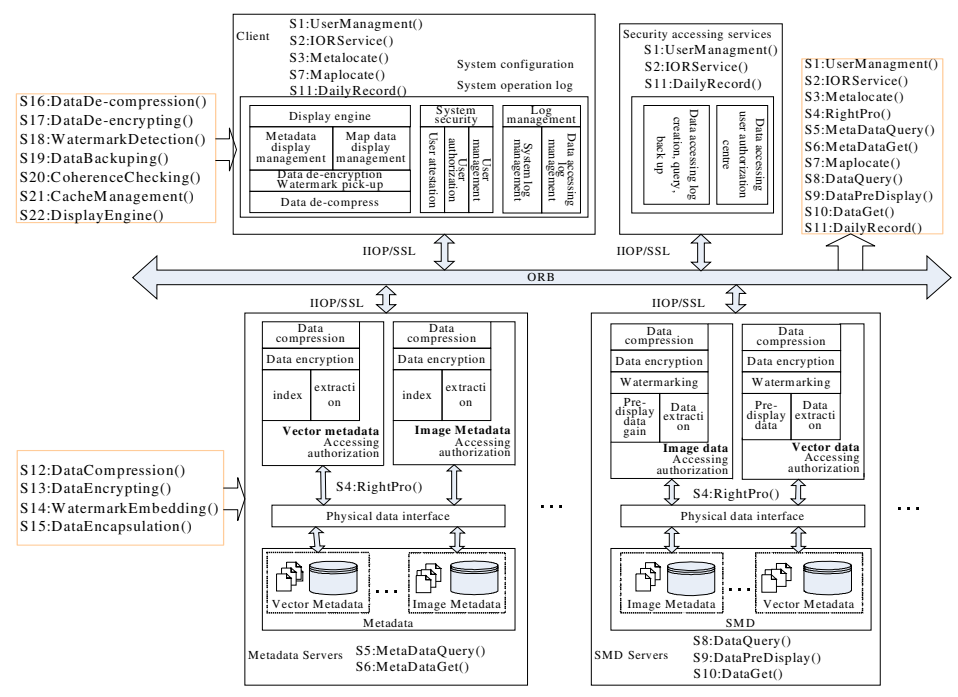

Fig. 1. Distributed CORBA services chart in MDND

\subsection{Realization of MDND}

From users' demands to object data, the operation steps are as follows:

(1) Demands logical presentation: There are three query methods related to $\mathrm{R}_{\text {sub }}^{1}$ to build correlative query condition $\alpha$, they are fulfilled based on catalog tree, visualization map window, and blurry restriction condition query.

(2) Metadata and SMD retrieving services: Compared with reference [16], this paper designed metadata centralization query service Metalocate() and SMD route 
pointing service Maplocate(). The searching procedure can be considered as a path in graph $\mathrm{G}$ from start resource node $\mathrm{n}_{0}$ to the node $\mathrm{n}_{\mathrm{i}}$. The dissemination flow shows as:

Dissemination (data type: Mvec, Bvec, Input: $\alpha$, Mvec,Bvec, Output: SMD-Vector Map)

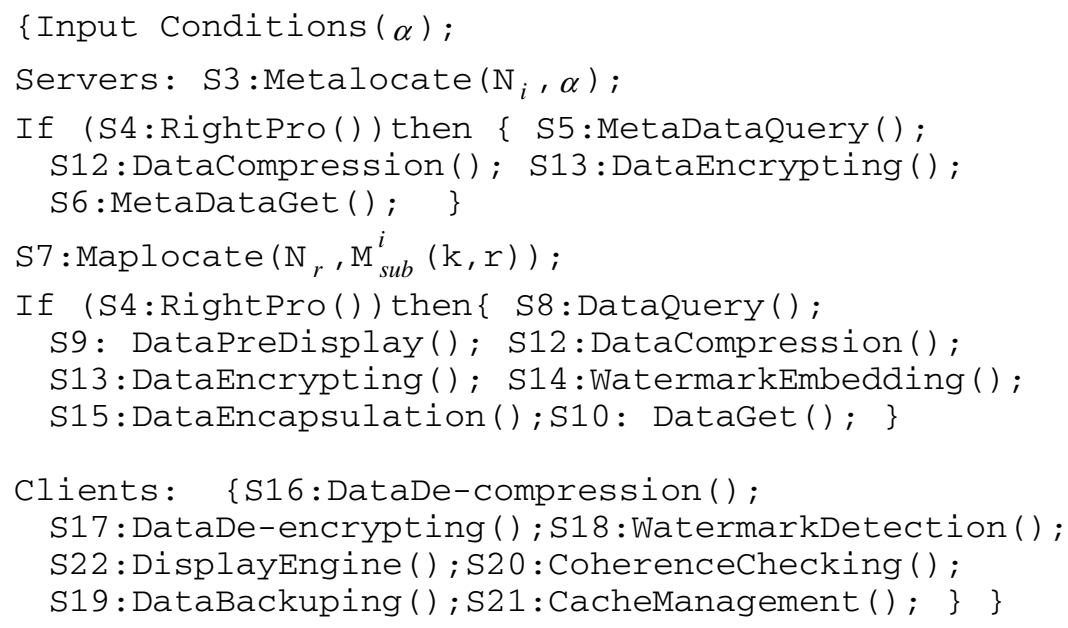

\section{Clients: $\quad$ S16:DataDe-compression( ) ;}

S17 : DataDe-encrypting ( ) ; S18:WatermarkDetection ( ) ;

S22 : DisplayEngine ( ) ; S2 0 : CoherenceChecking ( ) ;

S19:DataBackuping ();S21:CacheManagement (); \} \}

In the experiment, servers were installed in Xian Jiaotong University linked with internet, and the clients were set in Xian Research Institute. MDND is a user interactive course. In order to ensure veracity, we measured the time indexes three times by different presentation methods, and then calculated the average value.

We carried out a comparison study on transmitting performance between MDND and direct copy. Here, we used four vector map sheets of 1:50000 from Oracle database. The results are shown in Table 3. MDND can rapidly respond to network resources locating, especially for S3 and S7 services. A few instable performance indexes indicate that research institute intranet is not very steady. For mass image bigger than 100MB, transfer performance would attenuate because of repeated iterative algorithm.

Table 3. Transfer performance comparison

\begin{tabular}{lllll}
\hline Map ID & F2378 & F4765 & F15632 & F5634 \\
\hline Map data (MB) & 19.0 & 22.0 & 17.9 & 18.9 \\
S3:Metalocate() (ms) & 32 & 15 & 16 & 16 \\
S7:Maplocate() (ms) & 16 & 31 & 16 & 32 \\
S10:DataGet() (KB/s) & 573 & 450 & 498 & 525 \\
Copy (KB/s) & 626 & 513 & 564 & 567 \\
\hline
\end{tabular}

\section{Copyright Validating}

\subsection{Embedding and Extraction}

Embedding algorithm should explicitly make use of perceptually significant features in the data [17]. In the algorithm introduced here, the embedded intensity of 
watermark will be modulated adaptively according to the corresponding density of vertices. After the subdivision, according to the corresponding density of vertices, the embedded intensity of watermark in each block will be modulated adaptively as formula (5) and formula (6);

$$
\begin{gathered}
\alpha_{\mathrm{s}}=(1-\gamma) \times \alpha_{\max }+\gamma \times \alpha_{\text {min }} \\
\gamma=\left[\max \left(N P_{s}\right)-N P_{s}\right] /\left[\max \left(N P_{s}\right)-\min \left(N P_{s}\right)\right]
\end{gathered}
$$

$\alpha_{\mathrm{s}}(s=1,2, \cdots k * l)$ is the embedded intensity of rectangle blocks, $\alpha_{\max }$ is the maximum of embedded intensity, and $\alpha_{\min }$ is the minimum of embedded intensity. $\alpha_{\max }$ is chosen within the range of the maximal mapping error so that the displacement won't affect visual qualities of maps. $N P_{s}$ is the vertex numbers of rectangle blocks, $\max \left(N P_{s}\right)$ is the maximum of $N P_{s}$, and $\min \left(N P_{s}\right)$ is the minimum of $N P_{s}$.

According to the corresponding embedded intensity, the watermark is embedded into the vector map by displacing the vertices in the rectangles. Repeatedly, embedding each bit $\mathrm{c}$ times increases resiliency of the watermark against additive random noise. Formula (7) gives the method to compute c. NP is the number of vertices in the map.

$$
c=[N P / n]
$$

When extraction, different from other methods we compare the watermarked map with the original watermarked map, not with the primitive map. The original watermarked map, published to the users, is the unrevised watermarked map directly derived from the primitive map and has the same record order with the primitive map. If we align the watermarked map with the primitive map directly, it is very possible to make mistakes because there are two types of noises (watermark data and random noises) between the two types of map data. This bas been done by minimizing the Euclid distance between vertices in the watermarked and the original watermarked map.

When extracting the watermark image, we use mean-value detection, different from the double-threshold combinative detection used by Reference [15]. The algorithm can get the valid embedding times and the total embedding bits of each pixel in the two-value watermark image through the alignment and comparison mentioned. Then the mean value of the total embedding bits of each pixel can be easily computed and converted to the corresponding pixel value of the two-value watermarking image.

\subsection{Experiments and Results}

In the experiment, we used a 1:50000-scale vector map which has 10 feature layers and 380368 vertices, partly shown in Fig. 2, and its size is $9.85 \mathrm{MB}$. The two-value watermark image used here is a $128 \times 128 \mathrm{Bmp}$ file, shown in Fig. 2, and the size is 10.0 KB. The maximum of embedded intensity is set to $2.5 \mathrm{~m}$, and the minimum of embedded intensity is set to $1 \mathrm{~m}$. The map is divided into 30x3020 blocks. The watermark image was embedded into abscissa of vertices. 


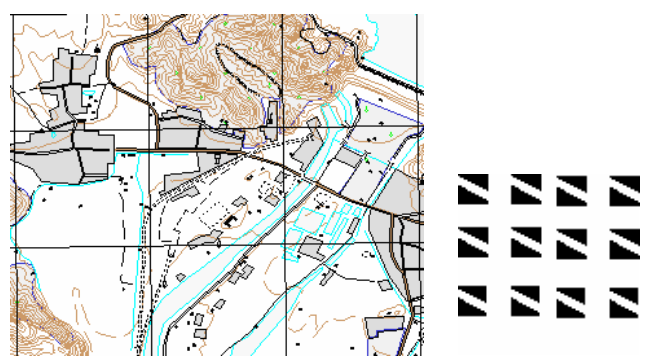

Fig. 2. Map and watermark image in experiment

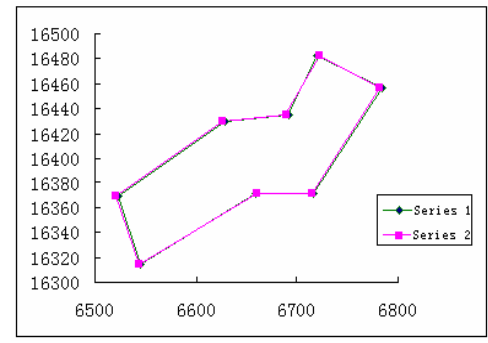

Fig. 3. Visual difference after zooming in

We compared the coordinate of watermarked map with the primitive map to observe the change of coordinate made by watermarking. The range of coordinate change is within $\pm 2 \mathrm{~m}$, and the watermarked result is satisfied with mapping demand. In Fig. 3, the visual difference between the two data is still very little after zooming in many levels. Here, Series 1 denotes primitive data and Series 2 denotes watermarked data.

Table 4 shows the results of the attack experiments under random noise, vertices insertion, vertices deletion, etc.. The advantage of the improved algorithm is especially presented in random noise of amplitude $28 \mathrm{~m}$. Fig. 4 gives the results of both algorithms against random noise attacks. (1) is the improved algorithm, (2) is [15] algorithm. The improved algorithm slightly outperformed the one in Reference [15] when we deleted 371527 vertices from the map data. From the experiments, we also found both algorithms withstand vertices insertion and random noise of amplitude $8 \mathrm{~m}$.

Table 4. Performance in various attacks

\begin{tabular}{cccc}
\hline Attacks & $\begin{array}{c}\text { Improved } \\
\text { algorithm }\end{array}$ & $\begin{array}{c}{[15]} \\
\text { algorithm }\end{array}$ \\
\hline Random & $8 \mathrm{~m}$ & 1.00 & 1.00 \\
noise & $28 \mathrm{~m}$ & 0.90 & 0.29 \\
vertices insertion & 1.00 & 1.00 \\
vertices deletion & 0.98 & 0.96 \\
\hline
\end{tabular}

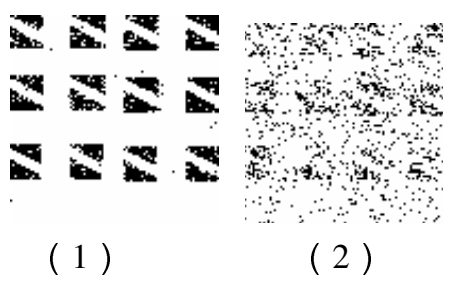

Fig. 4. Results of random noise attack

\section{Conclusion and Future Work}

The experiment results indicate that: (1)Remote user can get SMD conveniently with integrated SMD and network environment, which expands data application channel. (2) The services have excellent performances in metadata and SMD management, SMD location and accessing, and SMD transmitting, etc. (3) User is able to access SMD data in real time only after registration, authorization, request, download, and 
configuration. (4) The architecture is better for expanding servers, services, SMD resources, and overcomes other systems' disadvantage of bad expansibility. Furthermore, the system supports $\mathrm{C}++$ environment, complex SMD computation and mass data. At the same time, an improved watermarking algorithm for vector digital map presents the concept of original watermarked map in extraction. Experiments show that the improved algorithm is more resilient against many types of attacks.

In the future, we will study user dynamic customization technology and the redeveloping capability on the existing system.

\section{References}

1. Tsou, M., Buttenfield, B.: Client/Server components sand Metadata objects for distributed Geographic Information Services. In: Proceedings of GIS/LIS'98, November 1998, pp. 590-599 (1998)

2. Futrelle, J.: Developing metadata standards for science data reuse in NCSA's distributed Grid architecture, pp. 1217-1219. IEEE, Los Alamitos (2000)

3. Azman, I., Peter, T.: Data dissemination and pricing policy for spatial data at the Surveying and Mapping Authority of the republic of Slovenia. In: 8th EC-GI \& GIS work shop ESDI-A work in Progress Dublin, Ireland, July 2002, pp. 3-5 (2002)

4. Cromley, R.G., McGlamery, P.: Integrating spatial metadata and data dissemination over the internet. In: IASSIST Quarterly Spering, pp. 13-16 (2002)

5. Kobben, B.: Publishing maps on the web. In: Kraak, M.J., Brown, A. (eds.) Web Cartography, Taylor and Francis, New York (2001)

6. Wang, Y., Ge, L., Rizos, C., et al.: Spatial data sharing on GRID. Geomatics Research Australia (81), 3-18 (2004)

7. Zhang, D.: Map data visualization browser technology on network environment. Zhenzhou: Institute of Surveying and Mapping, Information Engineering University (2005)

8. Laszewski, G.v., Foster, I., Gawor, J., Lane, P.: A Java Commodity Grid Kit. In: Concurrency and Computation: Practice and Experience, vol. 13(8-9), pp. 643-662 (2001)

9. Wang, F.: A distributed Geographic Information System on the common object request broker Architecture (CORBA). Geoinformatic, 89-115 (2000)

10. Obelheiro, P.R., Fraga, J.S.: Role-based Access Control for CORBA Distributed Object System. In: Proceedings of the 7th International Workshop on Object-oriented Real-time Dependable Systems (2002)

11. Chung, H.-H., Chen, T., Kun, K.-S.: Watermarking 2D/3D Graphics for Copyright Protection. In: Proceedings of IEEE International Conference Acoustics, Speech, and Signal Processing, vol. 4, pp. 720-723 (2003)

12. Ohbuchi, R., Ueda, H., Endoh, S.: Robust Watermarking of Vector Digital Maps. In: Proc ICME2002, Lausanne, vol. 1, pp. 577-580. IEEE Computer Society Press, Los Alamitos (2002)

13. Nikolaidis, N., Pitas, I., Solachidis, V.: Fourier descriptors watermarking of vector graphics images. In: Proceedings of XIII Brazilian Symposium on Computer Graphics and Image Processing vol. 3, pp. 9-12 (2000)

14. Solachidis, V., Nikolaidis, N.: Watermarking Polygonal Lines Using Fourier Descriptors. In: IEEE 2000 ICASSP, pp. 1955-1958 (2000) 
15. LI, Y.-Y., XU, L.-P.: Copyright protection of the vector map using the digital watermark. Journal of Xidian University 31(5), 719-723 (2004)

16. Krauter, K., Buyya, R., Maheswarn, M.: A taxonomy and Survey of Grid resource management systems for distributed computing. In: Software Practice and Experience, vol. 32(2), pp. 135-164 (2002)

17. Kutter, M., Bhattacharjee, S.K., Ebrahimi, T.: Towards Second Generation Watermarking Schemes. In: IEEE 1999 ICIP, vol. 1, pp. 320-323 (1999) 